Apidologie, 1978, 9 (4), 263-272.

\title{
RYTHME CIRCADIEN DES ACIDES CÉTO-9 \\ DÉCÈNE-2 OÏQUE, PHÉROMONE DE LA REINE, ET HYDROXY-10 DÉCÈNE-2 OÏQUE DES OUVRIÈRES D'ABEILLES APIS MELLIFICA LIGUSTICA S.
}

\author{
Circadianer Rhythmus der 9-Keto-2-Decensäure, \\ des Pheromons der Königin, und der 10-Hydroxy-2-Decensäure \\ der Arbeitsbienen von Apis mellifica ligustica $S$.
}

\author{
Janine PAIN, Bernard ROGER \\ Station de Recherches sur l'Abeille et les Insectes Sociaux, \\ I.N.R.A., 91440 Bures Sur Yvette
}

\begin{abstract}
SUMMARY
CIRCADIAN RHYTHIM OF 9-KETODEC-2-ENOIC ACID, QUEEN PHEROMONE, AND 10-HYDROXYDEC-2-ENOIC ACID OF WORKER BEES, APIS MELIIFICA LIGUSTICA S.
\end{abstract}

During a period of 24 hours at the summer solstice, unmated queens 15 to 18 days old and attendant workers aged 17 to 18 days were taken and killed every three hours. Amounts of 9-ketodec-2-enoic acid of queens heads and those of 10 -hydroxydec-2-enoic acid of workers heads were investigated by gas chromatography.

The study of these two acids, one pheromonal ( 9 -ketodec-2-enoic acid), the other not pheromonal (10-hydroxydec-2-enoic acid) showed their production had important quantitative variations. In the queen, the production rhythm of the 9 -ketodec-2 enoic acid is unimodal and it is between 11 a.m. and 5 p.m. that the amount is the highest. In attendant workers the production rhythm of 10-hydroxydec-2enoic acid could be trimodal. Titrated amounts are high during both day and night.

These results are related with sexual activity of drones on the one hand, with a gland activity, characteristic of the worker bee when it is in presence of a queen, on the other hand.

\section{RÉSUMÉ}

Au cours d'une période de 24 heures au solstice d'été, des reines vierges àgées de 15 à 18 jours et des ouvrières accompagnatrices de 17 à 21 jours ont été prélevées et sacrifiées toutes les trois heures. Les teneurs en acide céto- 9 décène- 2 oĩque des têtes de reines et en acide hydroxy-10 décène- 2 oîque des têtes des ouvrières ont été examinées par la technique de la chromatographie en phase gazeuse. 
L'étude de ces deux acides, l'un phéromonal (acide céto-9 décène-2 ö̈que), l'autre non phéromonal (acide hydroxy-10 décène- 2 oïque) a montré que leur production présente des variations quantitatives importantes.

Chez la reine, le rythme de production de l'acide céto-9 décène-2 oïque est unimodal. C'est entre $11 \mathrm{~h}$ et $17 \mathrm{~h}$ que la quantité est la plus élevée.

Chez les ouvrières accompagnatrices, le rythme de production de l'acide hydroxy-10 décène-2 oïque pourrait être trimodal. Les quantités dosées sont importantes à la fois le jour et la nuit.

Ces résultats sont mis en relation d'une part avec l'activité sexuelle des mâles d'abeilles et d'autre part avec une activité glandulaire propre à l'ouvrière lorsqu'elle est en présence d'une reine.

Descripteurs : rythme circadien, acide céto- 9 décène- 2 oïque, acide hydroxy-10 décène- 2 oïque, phéromone royale, Apis mellifica ligustica, reine, ouvrière, insectes.

\section{INTRODUCTION}

On connaît depuis longtemps l'importance des rythmes biologiques qui règlent la plupart des processus vitaux.

Ces rythmes sont de différents types. Ceux qui nous intéressent dans ce travail concernent le comportement lorsque celui-ci est sous la dépendance des substances phéromonales.

Un certain nombre d'auteurs ont étudié les relations existant entre la production des phéromones sexuelles et le moment de la journée où celles-ci sont sécrétées par les insectes. Dans leur ensemble, ces travaux portent sur l'examen des réponses comportementales des mâles aux odeurs phéromonales attractives émises par les femelles au cours d'une période de 24 heures ou plus.

Les données les plus nombreuses à ce sujet sont apportées chez les lépidoptères. C'est la raisor. pour laquelle nous citerons un exemple choisi dans cet ordre : c'est en étudiant la petite tordeuse du thé (Adoxophyes fasciata), papillon diurne, que des auteurs japonais (K. NAGATA et al., 1972) ont trouvé que la concentration en phéromones des femelles vierges variait selon les différents moments de la journée. En fait, ce n'est pas la concentration en phéromone qu'ils évaluent directement mais l'activité du comportement d'accouplement des mâles lorsque ceux-ci répondent à la phéromone sexuelle des femelles. Leurs résultats précisent que la sécrétion phéromonale des papillons femelles non fécondés présente 2 maxima en 24 heures, le premier, le matin en fin de scotophase, et le deuxième à partir de midi.

Toutefois, chez d'autres espèces comme les Pyralides, il est noté que la phéromone sexuelle des femelles n'est pas produite à des heures bien définies (BARTH, 1937-1938). TrAynier (1970) remarque aussi que des extraits abdominaux de femelles Ephestia kühniella ne sont pas plus attractifs lorsqu'ils sont préparés à partir de femelles capturées le matin ou en fin de journée, que celles-ci soient ou non en posture d'appel.

Chez les Hyménoptères, peu de travaux ont été entrepris. JACOBSON (1972) cite le cas d'un braconide (Bracon hebetor) dont les femelles attirent les mâles au cours des 
heures matinales (Grosch, 1948) et d'une tenthrède (Diprion similis) qui attire son premier mâle vers 11 heures et dont l'activité sexuelle se poursuit jusqu'à 16 heures (Coppel et al. 1960).

Peu d'auteurs ont donc entrepris d'évaluer directement la quantité de phéromone sur une période de 24 heures, en effectuant le dosage dans les glandes ou d'autres parties du corps des insectes en élevage.

Dès 1970, nous nous sommes intéressés aux problèmes des rythmes biologiques (PAIN, Roger, 1970). En effet, nous avons mis en évidence une variation annuelle de la teneur en acide hydroxy-10 décène- 2 oïque dans les têtes d'ouvrières. Poursuivant nos travaux dans cette direction, nous avons démontré que les deux phéromones sexuelles contenues dans les têtes de reines (les acides céto- 9 et hydroxy-9 décène- 2 oïque) présentaient un rythme saisonnier très net. Les quantités les plus importantes apparaissaient au mois de juin. Or, dans le bassin parisien, le mois de juin constitue une période cruciale pour le développement de la colonie d'abeilles. C'est l'époque des fortes miellées mais aussi celle des accouplements et de l'essaimage. Nous avons constaté le parallélisme étroit qui existe entre le cycle annuel de production de l'acide céto-9 décène- 2 oïque et celui de l'acide hydroxy-10 décène- 2 oïque sécrété par les ouvrières et constituant essentiel de la gelée royale.

Nous avons donc été amenés à étudier les variations possibles de la teneur en acide céto- 9 décène- 2 oïque et hydroxy-10 décène- 2 oïque chez les reines et les ouvrières accompagnatrices au cours d'une période de 24 heures.

\section{MÉTHODES}

\section{Préparation du matériel biologique}

Nous avons utilisé des reines vierges issues d'un élevage expérimental. Celui-ci a été conduit dans les colonies d'Apis mellifica ligustica $\mathbf{S}$. d'un rucher couvert. Nous avons décidé de faire un élevage royal de façon à ce que les reines puissent naitre dans les premiers jours de juin. C'est à cette époque de l'année que des reines vierges encagées avec de petites populations d'ouvrières contiennent les quantités les plus importantes de phéromones royales : les acides céto-9 et hydroxy-9 décène- 2 oïque (PAIN, Roger, 1974). Nous avons déjà expliqué les rôles divers que jouent ces deux acides (PaIN, 1971).

Dès que les reines sont prêtes à naître, elles sont prélevées et introduites dans les cagettes PAIN (1966) contenant une trentaine d'ouvrières de 1 et 2 jours prélevées au hasard sur un cadre de couvain naissant. Après la naissance des reines, on complète la population jusqu'à 50 ouvrières avec des abeilles du même âge également prises au hasard.

Les abeilles reçoivent comme nourriture du candi, du pollen, stocké à l'état frais à $-25^{\circ} \mathrm{C}$, et de l'eau. Elles restent claustrées en étuve à $32^{\circ} \mathrm{C}$. Les reines séjournent 15 jours en milieu confiné à l'obscurité. Vers le quinzième jour de leur vie, on sait qu'elles produisent beaucoup d'acide céto- 9 décène- 2 oïque (PAin, Roger, 1976). Puis les reines sont prélevées au hasard par groupes de 10 toutes les 3 heures pendant une période de 24 heures. Ainsi, le premier groupe de reines a été sacrifié le 20 juin à 11 heures. Au total, nous avons utilisé 90 reines réparties en 9 groupes horaires. En attendant d'effectuer les dosages, les reines et les ouvrières accompagnatrices sont conservées dans un congélateur à $-25^{\circ} \mathrm{C}$. 


\section{Dosages des acides céto-9 et hydroxy-10 décène-2 oïque}

Le dosage de l'acide céto- 9 décène- 2 oïque a été effectué à partir des extraits céphaliques de reines vierges. Les têtes de reines ont été prélevées et groupées par 10 pour chaque série horaire et dosées globalement.

Connaissant le parallélisme biochimique qui existe entre la sécrétion de l'acide céto- 9 décène- 2 oïque et celle de l'acide hydroxy-10 décène- 2 oïque, nous avons dosé également ce dernier, contenu dans les têtes des ouvrières accompagnatrices.

Pour manipuler plus commodément les extraits céphaliques des ouvrières, nous avons choisi au hasard 50 abeilles dont on a prélevé les têtes. C'est à partir de ces 50 têtes d'ouvrières que nous avons préparé un extrait dans l'éther. La même opération a été répétée pour les huit autres séries horaires. Les dosages de l'acide hydroxy-10 décène-2 ö̈que correspondent donc à un extrait de 50 têtes d'ouvrières représentatif de 500 ouvrières accompagnatrices de 10 reines.

\section{Technique des dosages}

La technique de préparation des broyats de têtes de reines et de têtes d'ouvrières a déjà été décrite (Pain, Barbier, Roger, 1967 - Pain, Roger, 1970).

Pour mettre en évidence les acides céto- 9 et hydroxy - 10 décène- 2 oïque, nous avons utilisé la chromatographie en phase gazeuse. Les extraits sont obtenus à partir de broyats de têtes dans l'éther. Ils sont amenés à sec puis méthylés pour être repris par des quantités appropriées d'hexane.

Les acides céto- 9 et hydroxy- 10 décène- 2 oïque de synthèse servent de référence et les quantités utilisées pour le dosage des extraits de reines et d'ouvrières sont de l'ordre de $1 \mathrm{mg}$ et de $2 \mathrm{mg}$.

Nous avons utilisé un chromatographe gaz liquide à détection par ionisation de flamme (PaIN, Roger, TheurkaufF, 1974). Il comporte une colonne spiralée de $2 \mathrm{~m}$ en inox. Son diamètre est de 1/4 de pouce. La colonne est remplie de Kiselgur 80/100 mesh imprégné de P.E.G. à $15 \%$.

Nous avons effectué trois injections par extrait de têtes de reines et de têtes d'ouvrières. Les substances témoin de synthèse sont injectées entre chaque extrait céphalique.

Les dosages quantitatifs des extraits céphaliques sont obtenus par la comparaison des aires des pics d'enregistrement avec celles fournies par des échantillons d'esters méthyliques d'acide céto-9 et hydroxy10 dècène- 2 oïque de synthèse.

Le taux d'erreur est de l'ordre de $10 \%$ au cours d'une même série de mesures.

\section{RÉSULTATS}

\section{Résultats des dosages de l'acide céto-9 décène-2 oïque dans les têtes des reines vierges}

La figure 1 montre que les teneurs en acide céto- 9 décène- 2 oïque quantifiées dans les têtes de reines vierges âgées de 15 à 18 jours sont les plus faibles à 8 heures du matin $(270 \mu \mathrm{g})$. Puis en trois heures de temps, on s'aperçoit que la quantité d'acide céto-9 décène- 2 oïque a doublé. Les quantités les plus fortes se situent entre 11 et 17 heures. On peut considérer qu'à partir de 18 heures la quantité d'acide trouvée est de moins en moins importante. Elle reste étale entre 23 et 5 heures du matin, sans toutefois être négligeable $(355 \mu \mathrm{g})$.

Ces résultats indiquent que dans nos conditions expérimentales d'élevage et de claustration des abeilles, la teneur en acide céto- 9 décène- 2 oïque des têtes de reines 
vierges présente en juin un rythme unimodal accentué le jour et atténué la nuit jusqu'aux premières heures de la matinée.

\section{Résultats des dosages de l'acide hydroxy-10 décène-2 oïque dans les têtes des ouvrières accompagnatrices}

La figure 2 présente l'évolution quantitative de la teneur en acide hydroxy-10 décène- 2 oïque des têtes des ouvrières de 17 à 21 jours accompagnatrices des reines. On remarque que l'allure de la courbe ne se calque pas exactement sur celle de la figure 1. On note cependant une certaine concordance entre les deux maxima qui se situent exactement à la même heure ( 14 heures) pour les deux acides. Toutefois la comparaison s'arrête là car, avant et après 14 heures, la teneur en acide hydroxy-10 décène2 oïque est peu élevée alors que celle des reines reste importante.

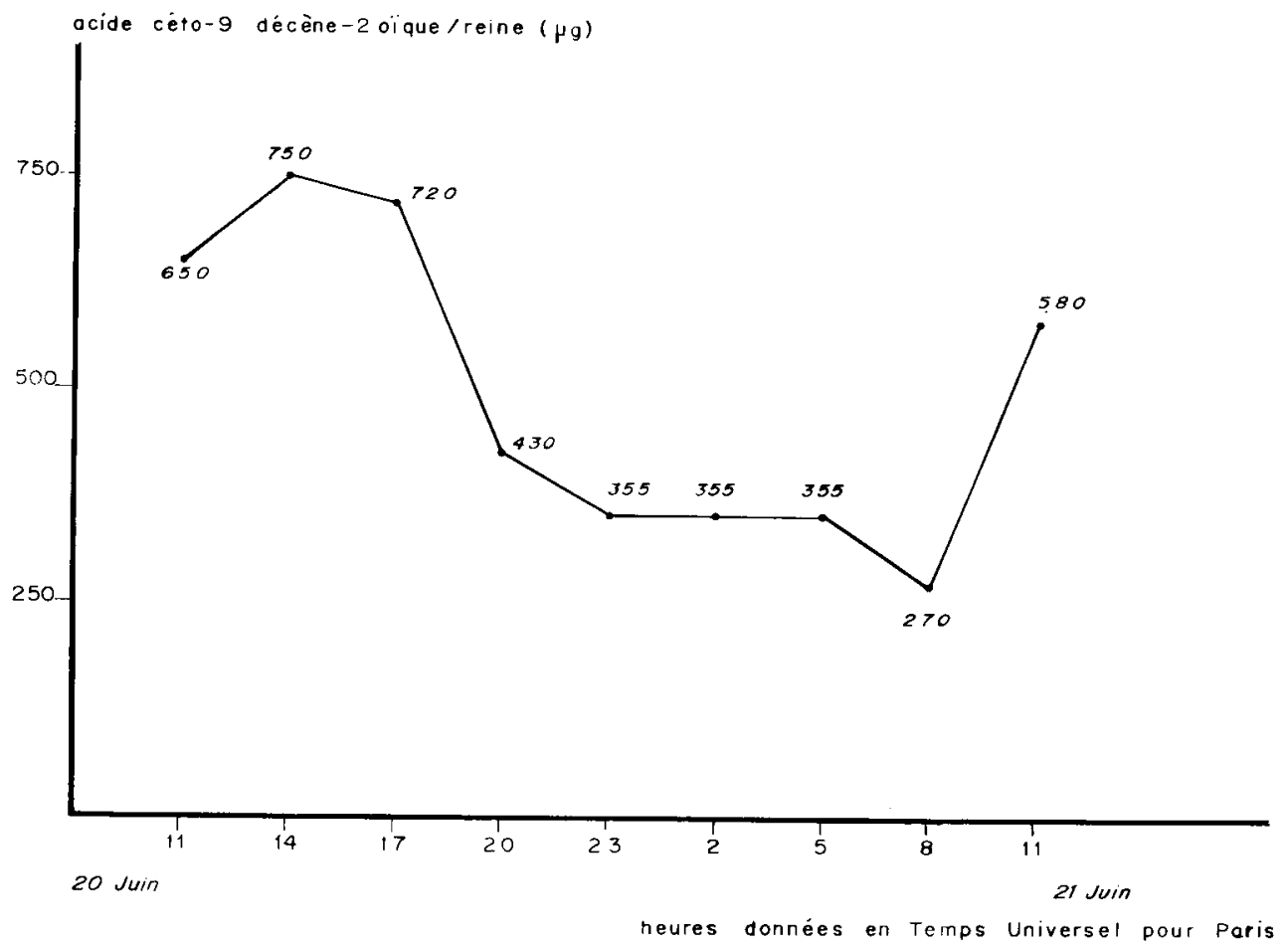

Fig. 1. - Quantité d'acide céto-9 décène-2 oïque $(\mu \mathrm{g})$ dans une tête de reine vierge de 15 à 18 jours (moyenne calculée sur 10 reines).

Les reines ont été prélevées toutes les 3 heures du 20 juin au 21 juin.

Aвв. 1. - Menge von 9-Keto-2-Decensäure $(\mu \mathrm{g})$ im Kopf einer jungfräulichen Königin im Alter von 15 - 18 Tagen (aus 10 Königinnen berechneter Mittelwert).

Die Königinnen wurden entnommen in Abständen von drei Stunden am 20. und 21. Juni. 


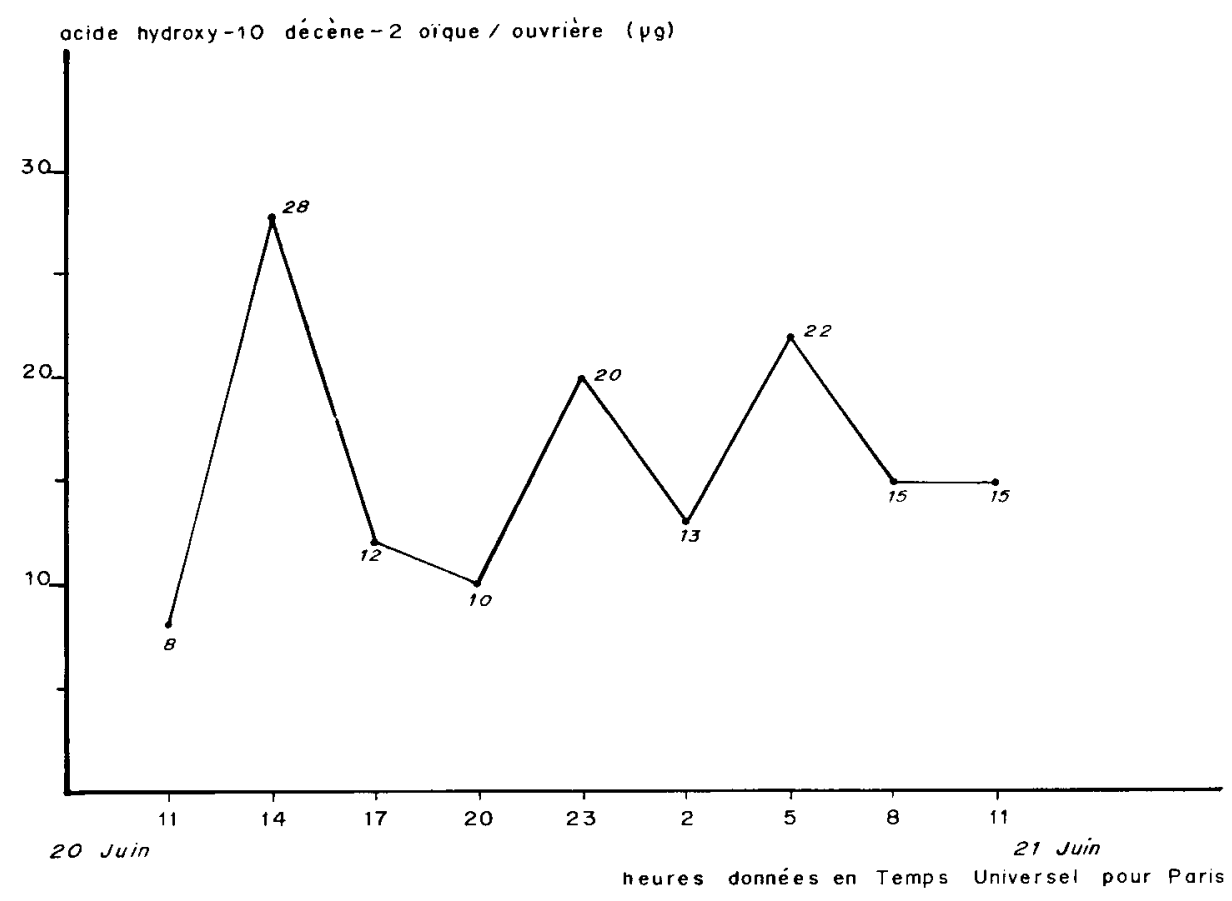

FIG. 2. - Quantité d'acide hydroxy-10 décène-2 oïque ( $\mu \mathrm{g})$ dans une tête d'ouvrière accompagnatrice de 17 à 21 jours (moyenne calculée sur 50 ouvrières).

Les ouvrières ont été prélevées toutes les 3 heures du 20 juin au 21 juin.

AвB. 2. - Menge von 10-Hydroxy-2-Decensäure ( $\mu \mathrm{g})$ im Kopf

einer Begleitarbeitsbiene, 17 - 21 Tage alt (aus 50 Arbeitsbienen berechneter Mittelwert).

Die Arbeitsbienen wurden in Abständen von drei Stunden am 20. und 21. Juni entnommen.

Chez les ouvrières, dès que la teneur en acide hydroxy-10 décène- 2 oïque a atteint son maximum, elle chute brusquement jusqu'à 17 heures pour s'abaisser encore jusqu'à 20 heures. Chez les reines, ce processus est plus lent puisque la teneur en acide céto-9 décène- 2 oïque diminue, rapidement jusqu'à 20 heures, plus doucement jusqu'à 23 heures.

On n'observe pas non plus comme chez les reines, une stabilité des quantités trouvées entre 23 et 5 heures du matin. Au contraire, l'activité des glandes céphaliques des ouvrières continue à se manifester au cours de la nuit.

Ces résultats montrent donc qu'au cours d'une période de 24 heures, l'acide hydroxy-10 décène- 2 oïque contenu dans les extraits céphaliques des ouvrières accompagnatrices présente au mois de juin une succession de rythmes d'une durée d'environ 6 heures, entrecoupée par des phases de repos ou de ralentissement. Chez les larves de drosophile RENSing (1969) a trouvé dans les glandes salivaires un rythme bimodal. 


\section{DISCUSSION}

Lors de travaux antérieurs (PAIN, RoGER, 1974) nous avons montré que l'acide céto-9 décène- 2 oïque présentait un rythme annuel et que celui-ci se calquait sur celui de l'acide hydroxy - 10 décène- 2 oïque, les quantités maxima des deux acides se situant en juin. Or, pour effectuer le dosage de ces deux acides, nous avons sacrifié les reines et les ouvrières dans la journée. Plus précisément, les reines ont été sacrifiées entre 10 et 17 heures et les ouvrières vers 14 heures. Ainsi, dans les deux cas, nous nous étions placés aux heures les plus favorables. Ce qui pourrait expliquer dans une faible mesure l'évolution parallèle des deux courbes.

Au cours d'autres recherches (PAIN, Roger, 1976) nous avons étudié la variation de la teneur en acide céto-9 décène- 2 oïque en fonction de l'âge des reines. Nous avons indiqué que la teneur en acide céto- 9 décène- 2 oïque est la plus forte chez des reines vierges âgées de 16 à 17 jours.

Le travail que nous venons d'effectuer chez les reines vierges de même âge, au cours d'une période de 24 heures au solstice d'été, fait apparaître de fortes quantités de phéromone (acide céto- 9 décène- 2 oïque). Toutefois, les valeurs trouvées bien qu'importantes restent en dessous de celles indiquées précédemment, établies en septembre (PAIN, Roger, 1976). Il est probable que les facteurs de l'environnement n'ont pas agi avec la même intensité sur la physiologie de la colonie au cours des deux années comparées.

En ce qui concerne les valeurs d'acide céto- 9 décène -2 oïque enregistrées chez les reines vierges, nous remarquons qu'elles sont les plus importantes aux heures les plus chaudes et les plus ensoleillées de la journée entre 11 et 17 heures. Il y a donc une analogie marquée avec le processus de l'accouplement. En effet, dans les conditions naturelles, les mâles d'abeilles s'accouplent avec les reines aux heures les plus chaudes et les plus ensoleillées qui correspondent aux maxima de la sécrétion céphalique des reines. En fin de journée, les quantités d'acide diminuent considérablement pour atteindre un palier qui reste stable 6 heures pendant lesquelles la teneur ne varie pas. Durant cette période, les valeurs trouvées $(355 \mu \mathrm{g})$ sont égales à environ la moitié des plus fortes $(750 \mu \mathrm{g})$. On peut supposer que pendant la nuit, l'acide céto-9 décène- 2 oïque aurait pour fonction de maintenir la cohésion des ouvrières autour de la reine. La chute de la quantité de substance que nous observons à 8 heures du matin pourrait s'expliquer par le fait qu'avant la reprise de la production phéromonale, les glandes auraient besoin d'un temps de repos avant de reprendre leur activité. Ainsi, le rythme circadien unimodal de la production d'acide céto- 9 décène- 2 oïque coïnciderait avec le rythme d'activité sexuelle des mâles.

En ce qui concerne la teneur en acide hydroxy-10 décène- 2 oïque des ouvrières accompagnatrices de reine, nous retrouvons en juin exactement les mêmes valeurs que celles que nous avions trouvées en 1970 (PAIN, RoGER). Dans nos conditions expérimentales, les reines n'ont pas pondu et les ouvrières n'ont pas élevé de couvain. Le 
rythme qui apparaît sur la figure 2 n'est donc pas en rapport avec une activité d'élevage. Mais puisque dès le premier jour de leur claustration, les jeunes ouvrières accompagnatrices ont consommé du pollen, elles ont donc reçu les éléments nutritifs nécessaires au développement des glandes céphaliques productrices de gelée. L'un de nous, B. RogER (G. ARNOLD, B. ROGER, sous presse) a montré l'effet accélérateur du pollen sur la production de l'acide hydroxy-10 décène- 2 oïque. Par conséquent, les quantités trouvées reflètent un rythme d'activité glandulaire propre à l'ouvrière lorsque celle-ci est en présence d'une reine. Ce rythme pourrait être trimodal. Il ne se calque pas sur celui de la teneur en acide céto- 9 décène- 2 oïque sauf au moment du maximum de la production, à 14 heures.

Nous pensons que ces variations dans la teneur en acide hydroxy-10 décène-2 oïque sont en rapport avec le nourrissage des reines par les ouvrières. De plus, il se pourrait que la reine influe aussi sur la teneur en acide hydroxy-10 décène- 2 oïque des ouvrières qui l'accompagnent.

\section{CONCLUSION}

Chez l'Abeille, c'est la première fois qu'un rythme circadien en rapport avec le contenu des glandes céphaliques des reines et des ouvrières a été découvert.

L'étude des deux acides, l'un phéromonal (l'acide céto- 9 décène- 2 oïque), l'autre non phéromonal (l'acide hydroxy - 10 décène- 2 oïque) a montré que leur production présente des variations quantitatives importantes au cours d'une période de 24 heures au solstice d'été.

Chez la reine, le rythme de production de l'acide céto- 9 décène- 2 oïque est de type unimodal. C'est le jour que la quantité la plus élevée est enregistrée, de 11 à 17 heures. Or, au cours de ces 6 heures, la phéromone émise par la reine déclenche l'activité sexuelle des mâles d'abeilles dont les vols de fécondation se situent sensiblement aux mêmes heures.

Chez les ouvrières accompagnatrices, le rythme de production de l'acide hydroxy10 décène- 2 oïque serait peut-être de type trimodal. Les quantités trouvées sont importantes à la fois le jour et la nuit. Le rythme observé chez les ouvrières se différencie de celui existant chez la reine, tout au moins en ce qui concerne l'augmentation des teneurs au cours de la nuit. La seule similitude entre le rythme de production des deux acides concerne le maximum des valeurs obtenues dans les deux cas, à 14 heures. Les différentes phases qui apparaissent en 24 heures sont sans doute liées à une activité glandulaire d'ordre alimentaire et biochimique.

D'autre part, les expériences ayant été réalisées avec des ouvrières de 17 à 21 jours, le rythme circadien de l'acide hydroxy-10 décène- 2 oïque correspond à celui d'abeilles d'âge homogène. Dans une colonie normale, les écarts d'âge sont plus grands (de 1 à 45 jours) et varient en fonction des saisons. 
L'observation des courbes permet de constater que les glandes céphaliques des reines et des ouvrières contiennent toujours des quantités d'acide mesurables. Cela signifie peut-être que les glandes ne se vident jamais complètement. Dans nos conditions expérimentales, on peut évaluer approximativement la quantité de phéromone produite par une reine vierge à $500 \mu \mathrm{g}$ par 24 heures. Cette quantité varie certainement selon l'état physiologique et l'âge de la reine, le nombre et l'âge des ouvrières accompagnatrices et la saison.

Nous conclurons en indiquant que ces résultats peuvent être mis à profit sur le plan des applications pratiques. En effet, si l'on désire procéder à des extractions biochimiques de la phéromone royale, il est rentable de prélever les reines à des heures bien précises de la journée pour obtenir le maximum de produit. Si l'on utilise les reines vivantes, il est préférable de tenir compte de l'heure à laquelle la quantité de substance est la plus grande afin de travailler avec des insectes dont le pouvoir d'attraction est maximum.

Ce travail représente une première approche de l'analyse des rythmes circadiens chez les abeilles.

\author{
Reçu pour publication en mai 1978 \\ Eingegangen im Mai 1978
}

\title{
ZUSAMMENFASSUNG
}

Die Untersuchung von zwei Säuren, von denen die eine als Pheromon wirkt (9-Keto-2-Decensäure), die andere nicht (10-Hydroxy-2-Decensäure), mittels Gaschromatographie hat zur Zeit der Sommersonnenwende beträchtliche quantitative Schwankungen ihrer Produktion im Verlauf von 24 Stunden ergeben.

Die Menge der Säuren wurde aus Kopfextrakten von unbegatteten Königinnen im Alter von 15-18 Tagen und aus den Köpfen von Begleit-Arbeitsbienen von Apis mellifica ligustica S. bestimmt. Die Königinnen waren zusammen mit 50 Arbeiterinnen 15 Tage lang gekäfigt. Dann wurden sie in Gruppen von 10 alle 3 Stunden während des Verlaufs von 24 Stunden getötet. Die Arbeiterinnen wurden zur Bestimmung der Dosis ebenfalls getötet.

Die Resultate weisen darauf hin, dass unter unseren Bedingungen der Aufzucht und der Haltung der Gehalt der Kopfextrakte unbegatteter Königinnen an 9-Keto-2-Decensäure im Juni einen unimodalen Rhythmus mit einer Erhöhung während des Tages und einer Verringerung während der Nacht bis in die frühen Morgenstunden aufweist. Es ist bekannt, dass die von der Königin abgegebenen Pheromone das Sexualverhalten der Drohnen auslösen, deren Flüge ja nur am Nachmittag stattfinden.

Bei den Begleitbienen zeigt die in den Kopfextrakten enthaltene 10-Hydroxy-2-Decensäure im Juni eine Folge von Rhythmen, Höhepunkte, unterbrochen von Phasen der Inaktivität oder der Abnahme. Die festgestellte Menge spiegelt einen eigenen Rhythmus der Drüsenaktivität der Arbeitsbienen, wenn sie sich in Gegenwart einer Königin befinden. Dieser Rhythmus scheint trimodal zu sein. Er deckt sich nicht mit dem Gehalt an 9-Keto-2-Decensäure, ausgenommen am Höhepunkt der Produktion, um $14 \mathrm{Uhr}$ im Monat Juni. 


\section{RÉFÉRENCES BIBLIOGRAPHIQUES}

BARTH R., 1937-1938. Herkunft, Wirkung und Eigenschaften des weiblichen Sexualduftstoffes einiger Pyraliden. Zool. Jahrb., Abt. Allg. Zool. Physiol. Tiere, 58, 297-329.

Coppel H. C., CASIDA J. E., Dauterman W. C., 1960. - Evidence for a potent sex attractant in the introduced pine sawfly, Diprion similis (Hymenoptera : Diprionidae). Ann. Entomol. Soc. Amer., 53, 510-512.

Grosch D.S., 1948. - Experimental Studies on the mating reaction of male Habrobracon. J. Comp. Physiol. Psychol., 41, 188.

Jacobson M., 1972. - Insect Sex Pheromones. Academic Press, New York and London, 161 p.

Nagata K., Tamaki Y., Noguchi H., Yushima T., 1972. - Changes in sex pheromone activity in adult females of the smaller Tea Tortrix moth Adoxophyes fasciata. J. Insect Physiol., 18, 339-346.

PAIN J., 1966. - Nouveau modèle de cagettes expérimentales pour le maintien d'abeilles en captivité. Ann. Abeille, 9 (1), 71-76.

Pain J., Barbier M., Roger B., 1967. - Dosages individuels des acides céto-9 décène-2 oïque et hydroxy-10 décène- 2 oïque dans les têtes des reines et des ouvrières d'abeilles. Ann. Abeille, 10 (1), 45-52.

Pain J., Roger B., 1970. - Variation annuelle de l'acide hydroxy-10 décène-2 oïque dans les têtes d'abeilles. Apidologie, 1 (1), 29-54.

Pain J., 1971. - Phéromones et comportement chez quelques hyménoptères. Apidologie, 2 (4), 319-355.

Pain J., Roger B., Theurkauff J., 1974. - Mise en évidence d'un cycle saisonnier de la teneur en acide céto- 9 et hydroxy- 9 décène- 2 oïque des têtes de reines vierges d'abeilles. Apidologie, 5 (4), 319-355.

PAIN J., Roger B., 1976. - Variation de la teneur en acide céto- 9 décène- 2 oïque en fonction de l'âge chez les reines vierges d'abeilles (Apis mellifica ligustica S.). C.R. Acad. Sci. Paris, 283, 797-799.

Rensing L., 1969. - Circadiane Rhythmik von Drosophila Speicheldrüsen in vivo, in vitro und nach Ecdysonzugabe. J. Insect. Physiol., 15, 12, 2285-2303.

TRAYNiER R. M. M., 1970. - Sexual behaviour of the Mediterranean flour moth, Anagasta kühniella : Some influences of age, photoperiod, and light intensity. Can. Entomol., 102, 534-540. 